\title{
Technical note: Validation of a handheld meter for measuring $\beta$-hydroxybutyrate concentrations in plasma and serum from dairy cows
}

\author{
A. Pineda and F. C. Cardoso ${ }^{1}$ \\ Department of Animal Sciences, University of Illinois, 1207 West Gregory Drive, Urbana 61801
}

\begin{abstract}
The use of handheld meters for cow-side tests for $\beta$-hydroxybutyrate (BHB) concentrations in whole blood has become common. The aim of this study was to compare serum BHB (sBHB) and plasma BHB (pBHB) concentrations analyzed using either a "gold standard" enzymatic laboratory method (LM; Randox Laboratories Ltd., Antrim, UK; cat. no. RB1007) or a handheld meter (PX; Precision Xtra, Abbott Diabetes Care Inc., Alameda, CA). Results from 374 (187 serum and 187 plasma) samples taken from Holstein cows from $11 \mathrm{~d}$ before ( 52 samples) to $5 \mathrm{~d}$ after parturition (137 samples) were used for the analysis. Statistical analysis was performed using the MIXED, REG, and LOGISTIC procedures of SAS (v9.4; SAS Institute Inc., Cary, NC). A linear mixed model with repeated measures was fitted for LM and PX. Regression and correlation analyses were completed to estimate the relationship and agreement between the 2 methods. Lin's concordance correlation coefficient (CCC) and Bland-Altman plots were used to evaluate agreement between LM and PX. Cross-validation by randomly splitting the data in model-building and validation sets was performed to estimate and validate the equation that predicted the LM results using PX. Receiver operating characteristic (ROC) curves were used to estimate the sensitivity (Se) and specificity (Sp) of PX at different threshold levels. The CCC was 0.74 for $\mathrm{pBHB}$ and 0.68 for sBHB. The $95 \%$ confidence interval of agreement of the Bland-Altman plot encompassed $96 \%$ of the difference between LM and PX for pBHB and $95 \%$ for sBHB. The mean difference for $\mathrm{pBHB}$ was $-0.50 \pm 0.25 \mathrm{mmol}$, and that for sBHB was $-0.63 \pm 0.41 \mathrm{mmol}$. The highest Se and Sp for PX were achieved when the threshold for ketosis was set to $1.8 \mathrm{mmol} / \mathrm{L}$ for $\mathrm{pBHB}$ and $2.1 \mathrm{mmol} / \mathrm{L}$ for sBHB. The area under the ROC curve was 0.97 for $\mathrm{pBHB}$ and 0.96 for sBHB. The negative bias shown by the Bland-Altman plots suggested that PX yielded
\end{abstract}

Received April 3, 2015.

Accepted August 30, 2015.

${ }^{1}$ Corresponding author: cardoso2@illinois.edu higher pBHB and sBHB concentrations than the LM. However, the excellent test characteristics and area under the ROC curve close to 1 indicated that PX at the adjusted thresholds was able to accurately classify between samples $<1.2 \mathrm{mmol} / \mathrm{L}$ and those $\geq 1.2 \mathrm{mmol} / \mathrm{L}$ based on the gold standard test.

Key words: $\beta$-hydroxybutyrate (BHB), dairy cow, ketosis, handheld meter

\section{Technical Note}

Extensive fat mobilization in peripartal dairy cows in negative energy balance leads to greater concentrations of circulating NEFA in blood and high ketone body (including BHB) production by the liver (Roche et al., 2013). Ketone bodies are utilized as an energy source but, during intense fat mobilization, production of ketone bodies can exceed utilization, leading to the development of ketosis. Two types of ketosis, subclinical and clinical, can be identified in cows. Subclinical ketosis is defined as an excess of circulating ketone bodies without clinical signs (Andersson, 1988).

The gold standard diagnostic test for ketosis is the measurement of BHB concentrations in plasma or serum due to its stability and predominance in whole blood compared with measuring acetone or acetoacetate under extensive fat mobilization (Oetzel, 2001). Serum or plasma BHB concentrations are commonly quantified by laboratories utilizing colorimetric enzymatic procedures. Recently, several authors have reported the association of blood BHB concentrations measured with handheld meters with standard methods in serum or plasma in dairy cows (Voyvoda and Erdogan, 2010; Iwersen et al., 2013), sheep (Panousis et al., 2012; Pichler et al., 2014b), goats (Doré et al., 2013; Pichler et al., 2014a), dogs and cats (Hoenig et al., 2008), and humans (Byrne et al., 2000), concluding that such devices, after threshold adjustment in some cases, were reliable on-site tests to measure BHB concentrations.

Most of the reported studies have compared wholeblood BHB concentrations determined with a handheld meter with serum or plasma BHB concentrations determined by using a gold standard procedure. In the field, 
accurate on-site tests are convenient for the diagnosis of ketosis. At times, sample availability or retrospective analysis might make it necessary or desirable to use the meter with serum or plasma samples. There is a lack of studies reporting BHB concentrations in serum or plasma determined with a handheld meter compared with those measured using laboratory methods. Therefore, the objective of our study was to compare serum BHB (sBHB) and plasma BHB (pBHB) concentrations analyzed using a handheld meter (PX; Precision Xtra, Abbott Diabetes Care Inc., Alameda, CA) and a laboratory method (LM; Randox Laboratories Ltd., Antrim UK; cat no. RB1007, as the gold standard), possibly reducing the cost and time associated with sample analysis.

Experimental procedures were approved by the University of Illinois Institutional Animal Care and Use Committee. Multiparous pregnant Holstein cows were selected during late lactation. After drying off, cows were housed in a ventilated enclosed barn with access to sand-bedded freestalls. When calving was imminent, cows were moved to a maternity box stall bedded deeply with wheat straw. During the dry period, cows were fed individually once a day at approximately $0630 \mathrm{~h}$. After parturition, cows were moved to a ventilated tiestall barn where they were individually fed once daily at approximately $0700 \mathrm{~h}$.

For the purpose of this study, 374 (187 serum and 187 plasma) samples were obtained from $11 \mathrm{~d}$ before (52 samples) to $5 \mathrm{~d}$ after parturition (137 samples). Samples of blood were obtained by puncture of coccygeal vein or artery with 20 -gauge $\times 2.5 \mathrm{~cm}$ needles (Becton Dickinson and Company, Franklin Lakes, NJ) 3 times weekly throughout the close-up dry period and once daily from parturition to $5 \mathrm{~d}$ after. Samples were collected within $1 \mathrm{~h}$ after feeding ( $\sim 0700 \mathrm{~h})$ into evacuated tubes for serum (SST; serum separator tube, containing clot activator) and plasma (K2 containing EDTA; Becton Dickinson and Company). Samples for plasma were placed on ice immediately after collection. Samples for serum were allowed to clot at room temperature for at least $30 \mathrm{~min}$ and then placed on ice. All tubes were centrifuged within $2 \mathrm{~h}$ of collection at $4^{\circ} \mathrm{C}$ for $15 \mathrm{~min}$ at $959 \times \mathrm{g}$. After centrifugation, aliquots of serum $(10$ $\mathrm{mL})$ and plasma $(10 \mathrm{~mL})$ were stored at $-20^{\circ} \mathrm{C}$ for 60 $\mathrm{d}$ until analysis. The concentration of BHB in serum and plasma was determined by 2 methods: (1) by the commercially available LM at the University of Illinois College of Veterinary Medicine Clinical Pathology laboratory, and (2) by using the PX handheld meter at the University of Illinois Department of Animal Sciences.

Statistical analysis was performed using the MIXED, REG, and LOGISTIC procedures of SAS (version 9.4,
SAS Institute Inc., Cary, NC). A linear mixed model with repeated measures was constructed to analyze concentration of BHB over time. The model contained the fixed effect of method. Cow was considered as a random effect and days relative to calving as a repeated measure. The covariance structures considered were compound symmetric, autoregressive order 1, and unstructured. The covariance structure that yielded the lowest Akaike value was used in the model (Littell et al., 1998). Degrees of freedom were estimated by using the Kenward-Roger method (Littell et al., 1998) in the model statement. To estimate and validate the equation that predicted the LM results using PX, cross-validation was performed by randomly splitting the data in model-building and validation sets. The model was utilized to predict the new BHB concentration in the validation set. Then, the mean of the squared prediction errors (MSPR) was calculated and compared with the mean squared error (MSE) yielded by regression fit to the model building set. For pBHB, the model-building set had 92 observations and the validation set had 91 samples. For sBHB, both sets had 91 observations. Residual distribution was evaluated for normality and homoscedasticity in the respective analyses.

Regression and correlation analyses were carried out to estimate the association between LM and PX. Correlation coefficient measured the strength of the relationship between LM and PX, not the agreement among them (Bland and Altman, 1986). Consequently, assessing a diagnostic test performance with correlation coefficients only may be inappropriate. Therefore, Bland-Altman plots (Bland and Altman, 1986) and Lin's concordance correlation coefficient (CCC; Crawford et al., 2007) were used to visualize and quantify, respectively, the agreement between the results from PX and LM.

Contingency $2 \times 2$ tables were created to obtain true-negative, true-positive, false-negative, and falsepositive values. These values were used to compute the test characteristics (sensitivity, specificity, and positive and negative predictive values). Sensitivity (Se) was calculated as the proportion of samples with BHB concentration $\geq 1.2 \mathrm{mmol} / \mathrm{L}$ correctly diagnosed as positive by PX. Specificity (Sp) was calculated as the proportion of samples with BHB concentration $<1.2 \mathrm{mmol} / \mathrm{L}$ correctly diagnosed as negative by PX. Positive predictive value $(+\mathbf{P V})$ was calculated as the proportion of the samples with positive test that were correctly diagnosed. Negative predictive value $(-\mathbf{P V})$ was calculated as the proportion of the samples with negative test that were correctly diagnosed. Receiver operating characteristic (ROC) curves were constructed to identify the threshold with PX that best discriminated 
between samples $<1.2 \mathrm{mmol} / \mathrm{L}$ and those $\geq 1.2 \mathrm{mmol} / \mathrm{L}$ based on the gold standard test. The area under the ROC curve (AUC) was used to assess the accuracy of the PX thresholds.

The objective of our study was to compare sBHB and pBHB concentrations analyzed using either a standard laboratory method (LM) or a handheld meter (PX). The PX generated higher $(P<0.01)$ pBHB $(1.45$ vs. $0.95 \mathrm{mmol} / \mathrm{L})$ and sBHB (1.63 vs. $1.00 \mathrm{mmol} / \mathrm{L})$ concentrations than LM (Figure 1). Consequently, adjustment of the thresholds was required for correct interpretation of the concentrations yielded by the handheld device. The ROC curves were developed to determine optimum thresholds, as well as to estimate the Se and Sp of PX at different threshold levels. The highest Se and Sp for PX were achieved when the threshold was set to $1.8 \mathrm{mmol} / \mathrm{L}$ for $\mathrm{pBHB}$ and $2.1 \mathrm{mmol} / \mathrm{L}$ for $\mathrm{sBHB}$ (Table 1). The threshold for pBHB had Se of $100 \%$ and Sp of $94 \%$, and the +PV and -PV were 82 and $100 \%$, respectively. The threshold for sBHB had Se of
$94 \%$ and $\mathrm{Sp}$ of $98 \%$, and the $+\mathrm{PV}$ and $-\mathrm{PV}$ were 94 and $98 \%$, respectively (Table 1). Other studies have reported high Se and Sp for determination of wholeblood BHB concentrations utilizing handheld meters. In dairy cows, Iwersen et al. (2009) found that PX had Se and Sp of 88 and $96 \%$ at $1.2 \mathrm{mmol} / \mathrm{L}$ of blood $\mathrm{BHB}$, respectively. When the threshold was raised to $1.4 \mathrm{mmol} / \mathrm{L}$, the Se and Sp increased to 96 and $97 \%$, respectively (Iwersen et al., 2009). The +PV were $82 \%$ for both thresholds, whereas -PV were 98 and $100 \%$ for 1.2 and $1.4 \mathrm{mmol} / \mathrm{L}$ of whole-blood BHB, respectively (Iwersen et al., 2009). Voyvoda and Erdogan (2010) found Se and Sp with a handheld meter of 85 and $94 \%$ at $1.2 \mathrm{mmol} / \mathrm{L}$ of blood BHB, respectively. When the threshold was increased to $1.4 \mathrm{mmol} / \mathrm{L}$, Se and Sp incremented to 90 and $98 \%$, respectively (Voyvoda and Erdogan, 2010). The +PV and -PV were 88 and $91 \%$ at a plasma BHB threshold of $1.2 \mathrm{mmol} / \mathrm{L}$ and 95 and $97 \%$ at $1.4 \mathrm{mmol} / \mathrm{L}$, respectively (Voyvoda and Erdogan, 2010). In dairy ewes, a handheld meter had high

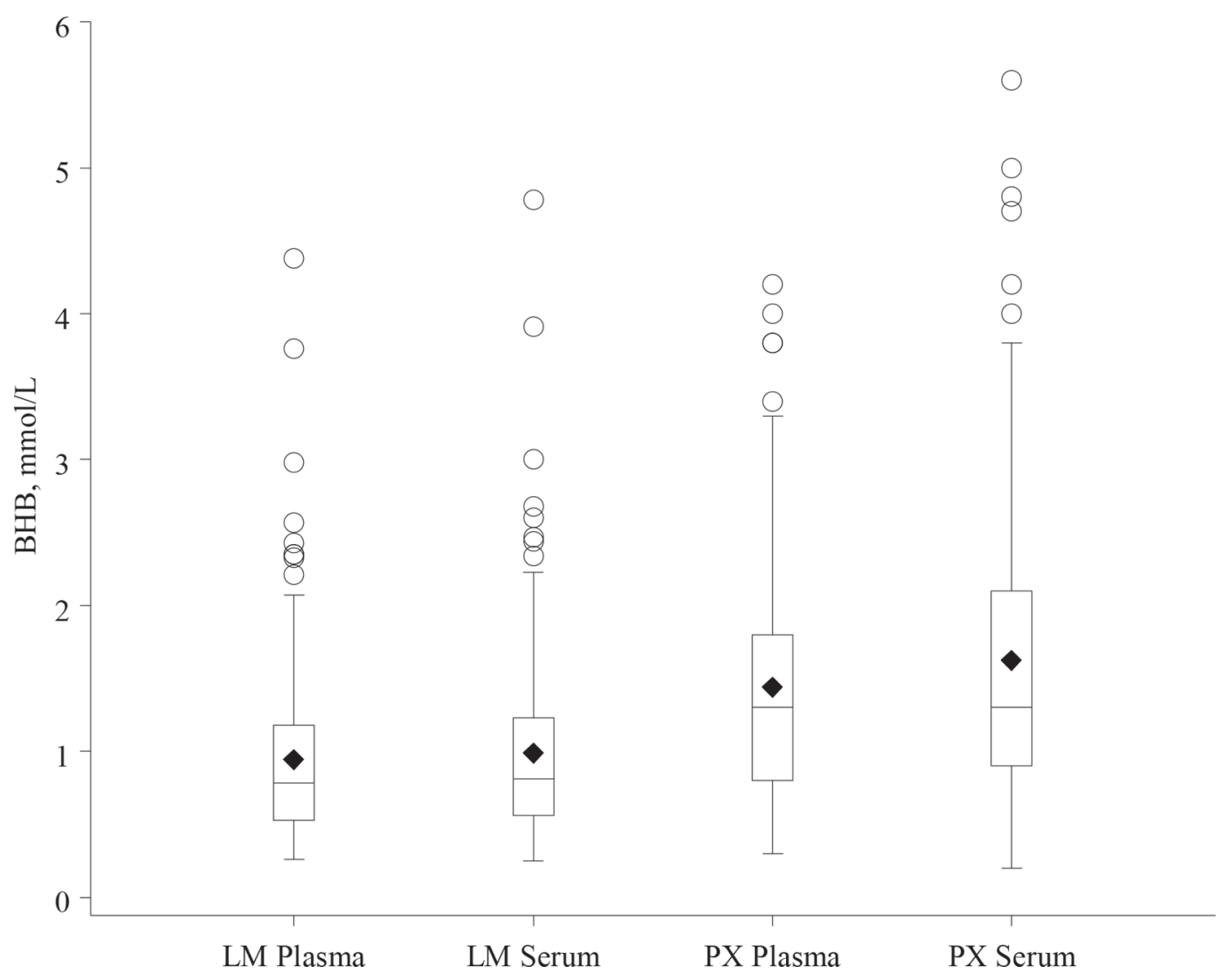

Figure 1. Box plot for plasma and serum BHB concentrations determined by a laboratory method (LM; gold standard) and a handheld meter (PX; Precision Xtra, Abbott Diabetes Care Inc., Alameda, CA). The black line inside each box presents the median (50th percentile), and lower and upper hinges mark the 25th and 75th percentiles, respectively. The filled diamond symbol within each box represents the group mean. Whiskers end at the smallest and largest statistical values that are not outliers; open circles indicate outliers (1.5 to 3.0 interquartile range). 
Table 1. Test characteristics for a handheld meter $^{1}$ at different BHB thresholds

PX BHB threshold (mmol/L)

\begin{tabular}{|c|c|c|c|c|c|c|c|c|c|c|c|c|}
\hline \multirow[b]{2}{*}{ Sample and characteristic } & & & & & & & & & & & & \\
\hline & 0.8 & 1.0 & 1.2 & 1.4 & 1.6 & 1.8 & 1.9 & 2.0 & 2.1 & 2.2 & 2.3 & 2.4 \\
\hline \multicolumn{13}{|l|}{ Plasma BHB $(\mathrm{n}=187)$} \\
\hline True negative, sample & 33 & 61 & 80 & 102 & 114 & 136 & 140 & 142 & 143 & 144 & 145 & 145 \\
\hline True positive, sample & 42 & 42 & 42 & 42 & 42 & 42 & 40 & 39 & 35 & 30 & 24 & 19 \\
\hline False negative, sample & 0 & 0 & 0 & 0 & 0 & 0 & 2 & 3 & 7 & 12 & 18 & 23 \\
\hline False positive, sample & 112 & 84 & 65 & 43 & 31 & 9 & 5 & 3 & 2 & 1 & 0 & 0 \\
\hline Positive predictive value, ${ }^{2} \%$ & 27 & 33 & 39 & 49 & 58 & 82 & 89 & 93 & 95 & 97 & 100 & 100 \\
\hline Negative predictive value, ${ }^{3} \%$ & 100 & 100 & 100 & 100 & 100 & 100 & 99 & 98 & 95 & 92 & 89 & 86 \\
\hline Sensitivity, ${ }^{4} \%$ & 100 & 100 & 100 & 100 & 100 & 100 & 95 & 93 & 83 & 71 & 57 & 45 \\
\hline Specificity, ${ }^{5} \%$ & 23 & 42 & 55 & 70 & 79 & 94 & 97 & 98 & 99 & 99 & 100 & 100 \\
\hline $\mathrm{AUC}^{6}$ & 0.61 & 0.71 & 0.78 & 0.85 & 0.89 & 0.97 & 0.96 & 0.95 & 0.95 & 0.85 & 0.79 & 0.73 \\
\hline \multicolumn{13}{|l|}{ Serum BHB ( $\mathrm{n}=187)$} \\
\hline True negative, sample & 36 & 56 & 70 & 98 & 106 & 119 & 126 & 133 & 134 & 137 & 137 & 137 \\
\hline True positive, sample & 50 & 50 & 50 & 50 & 50 & 50 & 48 & 47 & 47 & 44 & 41 & 38 \\
\hline False negative, sample & 0 & 0 & 0 & 0 & 0 & 0 & 2 & 3 & 3 & 6 & 9 & 12 \\
\hline False positive, sample & 101 & 81 & 67 & 39 & 31 & 18 & 11 & 4 & 3 & 0 & 0 & 0 \\
\hline Positive predictive value, $\%$ & 33 & 38 & 43 & 56 & 62 & 74 & 81 & 92 & 94 & 100 & 100 & 100 \\
\hline Negative predictive value, $\%$ & 100 & 100 & 100 & 100 & 100 & 100 & 98 & 98 & 98 & 96 & 94 & 92 \\
\hline Sensitivity, $\%$ & 100 & 100 & 100 & 100 & 100 & 100 & 96 & 94 & 94 & 88 & 82 & 76 \\
\hline Specificity, \% & 26 & 41 & 51 & 72 & 77 & 87 & 92 & 97 & 98 & 100 & 100 & 100 \\
\hline AUC & 0.63 & 0.70 & 0.76 & 0.86 & 0.89 & 0.93 & 0.94 & 0.96 & 0.96 & 0.94 & 0.91 & 0.88 \\
\hline
\end{tabular}

${ }^{1}$ Precision Xtra (PX; Abbott Diabetes Care Inc., Alameda, CA).

${ }^{2}$ Positive predictive value $=$ true positive $/($ true positive + false positive $)$.

${ }^{3}$ Negative predictive value $=$ true negative $/($ true negative + false negative $)$.

을 $\quad{ }^{4}$ Sensitivity $=$ true positive $/($ true positive + false negative $)$.

${ }^{5}$ Specificity $=$ true negative/(true negative + false positive).

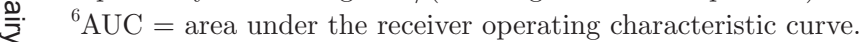


Se and Sp (98.6 and 98.2\%, respectively) as well as high $+\mathrm{PV}$ and $-\mathrm{PV}$ (99.3 and 96.4\%, respectively) at 0.8 $\mathrm{mmol} / \mathrm{L}$ of blood BHB (Panousis et al., 2012). The test characteristics in the present study are consistent with those reported in the aforementioned studies.

The AUC was used to assess the accuracy of the analyzed PX thresholds. When AUC $=0.5$, no discrimination exists; that is, the curve lies along the major diagonal line and the true- and false-positive proportions are equal (Swets, 1988). On the other hand, when AUC = 1.0, perfect discrimination exists, and the curve follows the left and upper axes such that the true-positive rate is 1.0 for all values of the false-positive rate (Swets, 1988). In our study, a greater AUC (0.97) for pBHB was achieved when the cutoff point was set to $1.8 \mathrm{mmol} / \mathrm{L}$. For sBHB, a greater AUC (0.96) was achieved when the cutoff point was set to 2.0 or $2.1 \mathrm{mmol} / \mathrm{L}$. However, at $2.1 \mathrm{mmol} / \mathrm{L}, \mathrm{PX}$ had identical Se and +PV (94\%) and identical Sp and -PV (98\%), and thus was accurately discriminating between samples with positive or negative test results. The LM was used as referent with a threshold of $1.2 \mathrm{mmol} / \mathrm{L}$ for subclinical ketosis. Iwersen et al. (2013) reported that a handheld meter had AUC of 0.99 and 1.0 at optimized device thresholds of 1.2 and $1.4 \mathrm{mmol} / \mathrm{L}$ of blood BHB, respectively. The corresponding Se and Sp were 98 and $90 \%$ at $1.2 \mathrm{mmol} / \mathrm{L}$ and 100 and $97 \%$ at $1.4 \mathrm{mmol} / \mathrm{L}$ of blood BHB, respectively. For a different device the AUC were 0.94 and 0.98 at optimized device thresholds of 1.1 and 1.3 $\mathrm{mmol} / \mathrm{L}$ of blood BHB, respectively (Iwersen et al., 2013). The corresponding Se and Sp were 80 and $87 \%$ at $1.1 \mathrm{mmol} / \mathrm{L}$ and 86 and $96 \%$ at $1.3 \mathrm{mmol} / \mathrm{L}$ of blood BHB, respectively. In dairy sheep, Pichler et al. (2014b) reported AUC of 0.96 and 0.99 at optimized handheld meter thresholds of 0.7 and $1.1 \mathrm{mmol} / \mathrm{L}$, respectively. The Se and Sp were 81.2 and $95.8 \%$ at $0.7 \mathrm{mmol} / \mathrm{L}$ and were 100 and $98.3 \%$ at $1.1 \mathrm{mmol} / \mathrm{L}$ of blood BHB (Pichler et al., 2014b).

The Bland-Altman method calculates the mean difference between 2 methods of measurement (the bias) and the $95 \%$ confidence interval of agreement as the mean difference $( \pm 1.96$ standard deviation). It is expected that the $95 \%$ confidence intervals of agreement include $95 \%$ of differences between the 2 measurement methods (Bland and Altman, 1986, 1999). Furthermore, a Bland-Altman plot that supports good agreement presents narrower limits of agreement, with differences between methods lying close along the mean difference, which ideally is close to zero. In the present study, the Bland-Altman plot for pBHB showed that the $95 \%$ confidence interval of agreement $(-1.00$ to $-0.004 \mathrm{mmol})$ encompassed $96 \%(179 / 187)$ of the difference between LM and PX. The mean difference between pBHB deter- mined by LM and PX was $-0.50 \mathrm{mmol}$, with a standard deviation of $0.25 \mathrm{mmol}$ (Figure 2A). For sBHB, the Bland-Altman plot revealed that the $95 \%$ confidence interval of agreement $(-1.45$ to $-0.19 \mathrm{mmol})$ included 95\% (176/186) of the difference between LM and PX. The mean difference between sBHB determined by LM and PX was $-0.63 \mathrm{mmol}$, with a standard deviation of $0.41 \mathrm{mmol}$ (Figure 2B). The $\mathrm{CCC}$ is a standardized coefficient that takes values between -1 and 1 , where -1 means perfect disagreement, 0 indicates an independent situation, and 1 means perfect agreement (Carrasco et al., 2013). In our study, the CCC were 0.74 and 0.68 for $\mathrm{pBHB}$ and $\mathrm{sBHB}$, respectively. Although the CCC showed fair agreement between LM and PX, and the differences between both methods fell within the $95 \%$ confidence interval of agreement, significant negative bias was observed in the Bland-Altman plot for $\mathrm{pBHB}$ and sBHB. For both $\mathrm{pBHB}$ and sBHB, the plot showed a wider variation with increasing BHB concentrations. Several studies that evaluated handheld meters for measurement of blood BHB concentration reported high Pearson (Iwersen et al., 2009; Voyvoda and Erdogan, 2010; Panousis et al., 2012) and Spearman (Doré et al., 2013; Iwersen et al., 2013; Pichler et al., 2014b) correlation coefficients, although these assess the relationship, not the agreement, between 2 methods. In our study, the Pearson correlation coefficients between LM and PX for pBHB and sBHB were 0.985 and 0.989 , respectively (Figure 3 ), in agreement with the previous studies; however, the CCC showed just fair agreement. Some studies reported high BlandAltman plot agreement (Iwersen et al., 2009, 2013), whereas others reported only fair agreement (Voyvoda and Erdogan, 2010; Pichler et al., 2014a) between a gold standard method and handheld meters for determination of blood BHB concentration.

Most studies have considered the relationship between BHB concentrations in whole blood and BHB concentrations in serum or plasma from laboratory methods, the last being considered the gold standard in all cases. In the present study, the models obtained from the building set that predicted LM concentrations using PX were $y=-0.05353+0.67842 x$ for plasma and $y=0.05542+0.56862 x$ for serum (Figure 2 ), where $y=$ predicted LM BHB and $x=\mathrm{PX}$ BHB. The MSE were 0.006 and 0.005 , whereas the MSPR were 0.007 and 0.006 for $\mathrm{pBHB}$ and sBHB, respectively. The MSPR was similar to MSE, which indicated that the equation generated from the regression models was not biased and gave an appropriate indication of the predictive ability of the models (Kutner et al., 2004).

In conclusion, the negative bias showed by the Bland-Altman plot suggested that $\mathrm{pBHB}$ and sBHB 

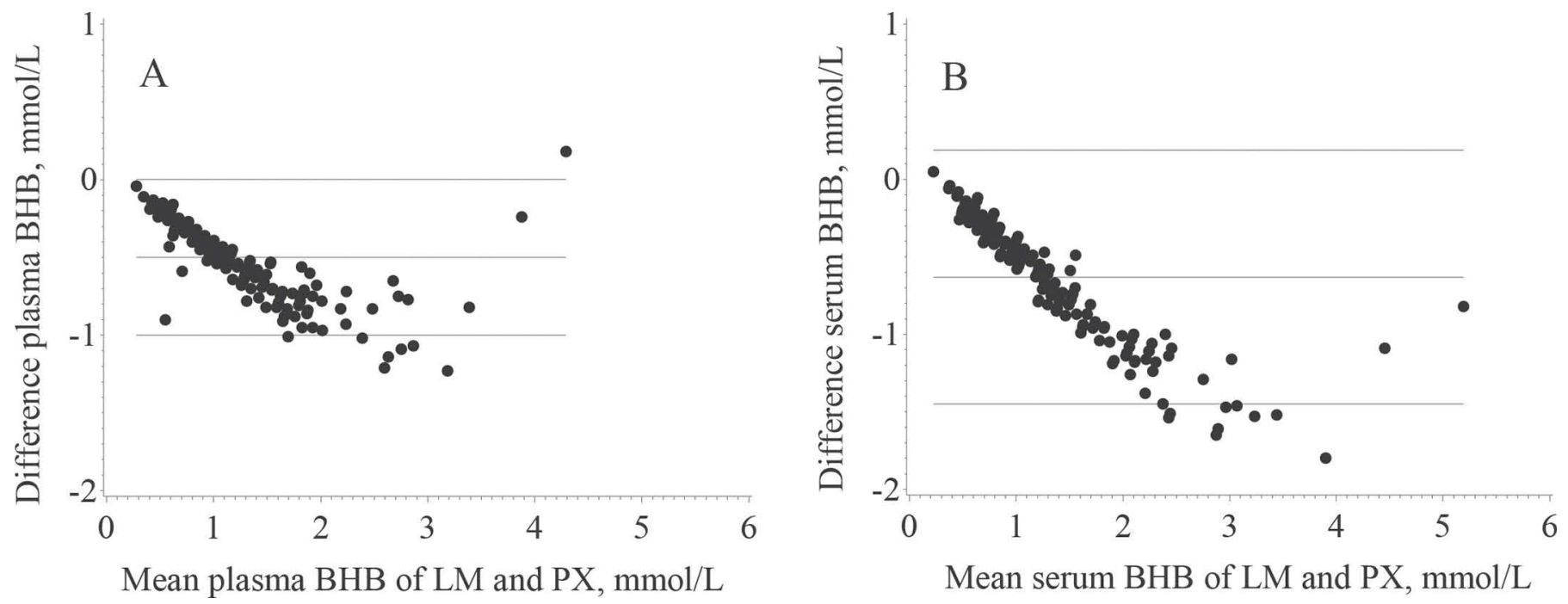

Figure 2. Bland-Altman plot of differences between (A) plasma and (B) serum BHB concentrations measured at the laboratory as determined by an enzymatic laboratory method (LM; gold standard) and a handheld meter (PX; Precision Xtra, Abbott Diabetes Care Inc., Alameda, CA) plotted against their mean concentrations. The solid line in the middle represents the mean; the upper and lower lines represent the mean $\pm 2 \mathrm{SD}$.

concentrations measured with the PX handheld meter led to higher BHB concentration values than the LM. However, the excellent test characteristics and AUC close to 1 indicated that, at the adjusted threshold values, PX was able to accurately discriminate between samples $<1.2 \mathrm{mmol} / \mathrm{L}$ and those $\geq 1.2 \mathrm{mmol} / \mathrm{L}$ based on the gold standard test. The handheld meter can be

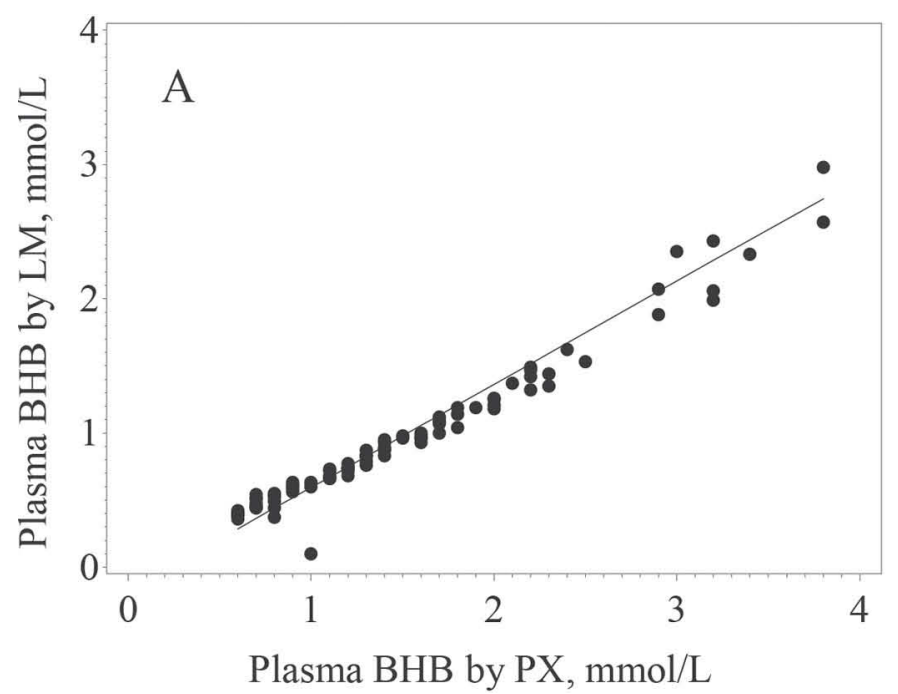

Figure 3. Regression plot for the model building set that predicted BHB concentrations as determined by an enzymatic laboratory method (LM; gold standard) and a handheld meter (PX; Precision Xtra, Abbott Diabetes Care Inc., Alameda, CA). A) Plasma BHB concentrations, adjusted $\mathrm{R}^{2}=0.98, \mathrm{r}=0.99, P<0.0001, y=-0.05353+0.67842 x ;(\mathrm{B})$ serum $\mathrm{BHB}$ concentrations, adjusted $\mathrm{R}^{2}=0.98, \mathrm{r}=0.99, P<0.0001$, $y=0.05542+0.56862 x$. For both models, $y=$ predicted LM BHB and $x=$ PX BHB.

an economical and viable method to determine plasma and serum BHB concentrations in dairy cows.

\section{ACKNOWLEDGMENTS}

The authors acknowledge the USDA-Section 1433 Animal Health and Disease (AHD) Research Funds,

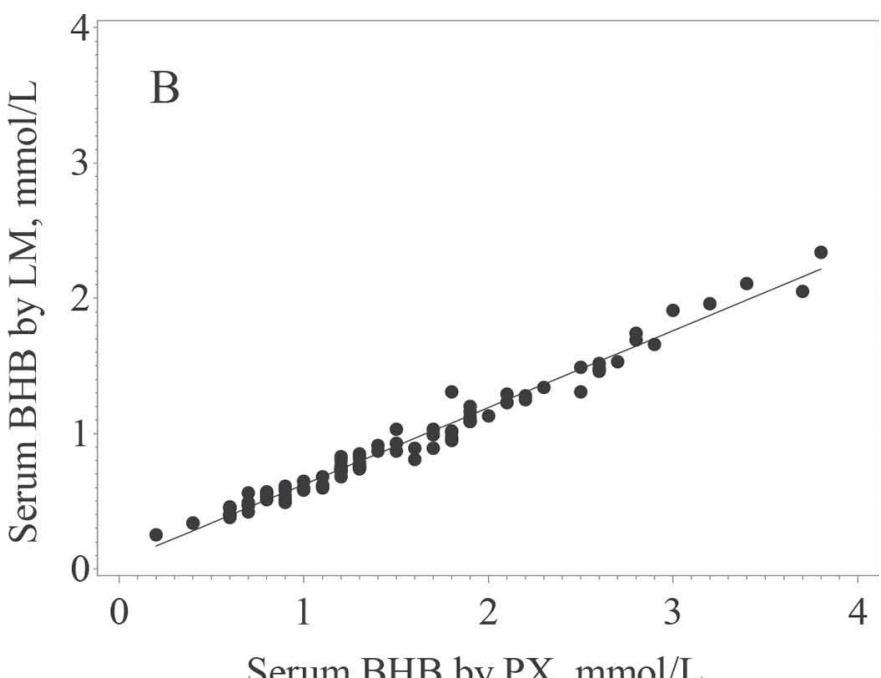

Serum BHB by PX, mmol/L 
project number: ILLU 538-935, for the financial support to carry out this experiment.

\section{REFERENCES}

Andersson, L. 1988. Subclinical ketosis in dairy cows. Metabolic diseases of ruminant livestock. Vet. Clin. North Am. Food Anim. Pract. 4:233-251.

Bland, J. M., and D. G. Altman. 1986. Statistical methods for assessing agreement between two methods of clinical measurement. Lancet 1:307-310.

Bland, J. M., and D. G. Altman. 1999. Measuring agreement in method comparison studies. Stat. Methods Med. Res. 8:135-160.

Byrne, H. A., K. L. Tieszen, S. Hollis, T. L. Dornan, and J. P. New. 2000. Evaluation of an electrochemical sensor for measuring blood ketones. Diabetes Care 23:500-503.

Carrasco, J. L., B. R. Phillips, J. Puig-Martinez, T. S. King, and V. M. Chinchilli. 2013. Estimation of the concordance correlation coefficient for repeated measures using SAS and R. Comput. Methods Programs Biomed. 109:293-304.

Crawford, S. B., A. S. Kosinski, H. M. Lin, J. M. Williamson, and H. X. Barnhart. 2007. Computer programs for the concordance correlation coefficient. Comput. Methods Programs Biomed. 88:62-74.

Doré, V., J. Dubuc, A. M. Bélanger, and S. Buczinski. 2013. Short communication: Evaluation of the accuracy of an electronic onfarm test to quantify blood $\beta$-hydroxybutyrate concentration in dairy goats. J. Dairy Sci. 96:4505-4507.

Hoenig, M., M. Dorfman, and A. Koenig. 2008. Use of a hand-held meter for the measurement of blood beta-hydroxybutyrate in dogs and cats. J. Vet. Emerg. Crit. Care 18:86-87.

Iwersen, M., U. Falkenberg, R. Voigtsberger, D. Forderung, and W. Heuwieser. 2009. Evaluation of an electronic cowside test to detect subclinical ketosis in dairy cows. J. Dairy Sci. 92:2618-2624.
Iwersen, M., D. Klein-Jöbstl, M. Pichler, L. Roland, B. Fidlschuster, I Schwendenwein, and M. Drillich. 2013. Comparison of 2 electronic cowside tests to detect subclinical ketosis in dairy cows and the influence of the temperature and type of blood sample on the test results. J. Dairy Sci. 96:7719-7730.

Kutner, M. H., C. J. Nachtsheim, J. Neter, and W. Li. 2004. Building the regression model I: model selection and validation. Pages 343383 in Applied Linear Statistical Models. 5th ed. McGraw Hill.

Littell, R. C., P. R. Henry, and C. B. Ammerman. 1998. Statistical analysis of repeated measures data using SAS procedures. J. Anim. Sci. 76:1216-1231.

Oetzel, G. R. 2001. Herd-based biological testing for metabolic disorders. American Association of Bovine Practitioners, 34th Annual Convention, Vancouver, BC. Am. Assoc. Bovine Pract., Auburn, AL.

Panousis, N., C. Brozos, I. Karagiannis, N. D. Giadinis, S. Lafi, and M. Kritsepi-Konstantinou. 2012. Evaluation of Precision Xceed ${ }^{\circledR}$ meter for on-site monitoring of blood $\beta$-hydroxybutyric acid and glucose concentrations in dairy sheep. Res. Vet. Sci. 93:435-439.

Pichler, M., A. Damberger, T. Arnholdt, I. Schwendenwein, J. Gasteiner, M. Drillich, and M. Iwersen. 2014a. Evaluation of 2 electronic handheld devices for diagnosis of ketonemia and glycemia in dairy goats. J. Dairy Sci. 97:7538-7546.

Pichler, M., A. Damberger, I. Schwendenwein, J. Gasteiner, M. Drillich, and M. Iwersen. 2014b. Thresholds of whole-blood $\beta$-hydroxybutyrate and glucose concentrations measured with an electronic hand-held device to identify ovine hyperketonemia. J. Dairy Sci. 97:1388-1399.

Roche, J., A. Bell, T. Overton, and J. Loor. 2013. Nutritional management of the transition cow in the 21st century-A paradigm shift in thinking. Anim. Prod. Sci. 53:1000-1023.

Swets, J. A. 1988. Measuring the accuracy of diagnostic systems. Science 240:1285-1293.

Voyvoda, H., and H. Erdogan. 2010. Use of a hand-held meter for detecting subclinical ketosis in dairy cows. Res. Vet. Sci. 89:344-351. 